\title{
Genomics of glycopeptidolipid biosynthesis in Mycobacterium abscessus and $M$. chelonae
}

\author{
Fabienne Ripoll ${ }^{1}$, Caroline Deshayes ${ }^{2,3}$, Sophie Pasek ${ }^{4}$, Françoise Laval ${ }^{5}$, \\ Jean-Luc Beretti ${ }^{3}$, Franck Biet ${ }^{6}$, Jean-Loup Risler ${ }^{4}$, Mamadou Daffé ${ }^{5}$, \\ Gilles Etienne ${ }^{5}$, Jean-Louis Gaillard ${ }^{1}$ and Jean-Marc Reyrat*2,3
}

Address: ${ }^{1}$ Laboratoire de Microbiologie, Université de Versailles Saint-Quentin-en-Yvelines, Faculté de Médecine de Paris-Ile de France-Ouest, F92380 Garches, France, 2Inserm-UMR 570, Unité de Pathogénie des Infections Systémiques, Groupe Avenir, Paris Cedex 15, F-75730, France, ${ }^{3}$ Université Paris Descartes, Faculté de Médecine René Descartes, Paris Cedex 15, F-75730, France, ${ }^{4}$ Laboratoire Génome et Informatique, UMR CNRS 8116, F-91034 Evry cedex, France, ${ }^{5}$ Institut de Pharmacologie et Biologie Structurale (UMR 5089), Département "Mécanismes Moléculaires des Infections Mycobactériennes", 205, route de Narbonne, F-31077 Toulouse-cedex, France and "INRA-Tours. UR918, F-37380 Nouzilly, France

Email: Fabienne Ripoll - ripoll.f@libertysurf.fr; Caroline Deshayes - deshayes@necker.fr; Sophie Pasek - sophie.pasek@genopole.cnrs.fr; Françoise Laval - Francoise.Laval@ipbs.fr; Jean-Luc Beretti - beretti@necker.fr; Franck Biet - Franck.Biet@tours.inra.fr; JeanLoup Risler - risler@genopole.cnrs.fr; Mamadou Daffé - daffe@ipbs.fr; Gilles Etienne - gilles.etienne@ipbs.fr; Jean-Louis Gaillard - jeanlouis.gaillard@apr.ap-hop-paris.fr; Jean-Marc Reyrat* - reyrat@ necker.fr

* Corresponding author

Published: 9 May 2007

BMC Genomics 2007, 8:1/4 doi:|0.||86/|47|-2|64-8-I|4
Received: 22 November 2006

Accepted: 9 May 2007

This article is available from: http://www.biomedcentral.com//47/-2/64/8/II4

(c) 2007 Ripoll et al; licensee BioMed Central Ltd.

This is an Open Access article distributed under the terms of the Creative Commons Attribution License (http://creativecommons.org/licenses/by/2.0), which permits unrestricted use, distribution, and reproduction in any medium, provided the original work is properly cited.

\begin{abstract}
Background: The outermost layer of the bacterial surface is of crucial importance because it is in constant interaction with the host. Glycopeptidolipids (GPLs) are major surface glycolipids present on various mycobacterial species. In the fast-grower model organism Mycobacterium smegmatis, GPL biosynthesis involves approximately 30 genes all mapping to a single region of $65 \mathrm{~kb}$.
\end{abstract}

Results: We have recently sequenced the complete genomes of two fast-growers causing human infections, Mycobacterium abscessus (CIP I04536T) and M. chelonae (CIP I04535T). We show here that these two species contain genes corresponding to all those of the M. smegmatis "GPL locus", with extensive conservation of the predicted protein sequences consistent with the production of GPL molecules indistinguishable by biochemical analysis. However, the GPL locus appears to be split into several parts in $M$. chelonae and $M$. abscessus. One large cluster (19 genes) comprises all genes involved in the synthesis of the tripeptide-aminoalcohol moiety, the glycosylation of the lipopeptide and methylation/acetylation modifications. We provide evidence that a duplicated acetyltransferase (atfl and atf2) in M. abscessus and $M$. chelonae has evolved through specialization, being able to transfer one acetyl at once in a sequential manner. There is a second smaller and distant (M. chelonae, $900 \mathrm{~kb} ;$ M. abscessus, $3 \mathrm{Mb}$ ) cluster of six genes involved in the synthesis of the fatty acyl moiety and its attachment to the tripeptide-aminoalcohol moiety. The other genes are scattered throughout the genome, including two genes encoding putative regulatory proteins.

Conclusion: Although these three species produce identical GPL molecules, the organization of GPL genes differ between them, thus constituting species-specific signatures. An hypothesis is that the compact organization of the GPL locus in $M$. smegmatis represents the ancestral form and that evolution has scattered various pieces throughout the genome in $M$. abscessus and $M$. chelonae. 


\section{Background}

Mycobacterium abscessus and $M$. chelonae are both species of rapidly growing mycobacteria (RGM) that have emerged as significant pathogens in humans during the last ten years: both species are major causes of skin and soft tissue infections following medical or surgical procedures [1]; M. abscessus also causes pulmonary infections and is increasingly recovered from patients with cystic fibrosis [1]. M. chelonae and M. abscessus are among the most-antibiotic resistant RGM species [1] and this has serious consequences for therapy [2].

Glycopeptidolipids (GPLs) are surface lipids found on a number of mycobacterial species including both RGM (e.g., M. smegmatis, M. chelonae, M. abscessus) and slow growing mycobacteria (e.g., M. avium subsp. avium). These molecules can make up more than $70 \%$ of the lipids exposed at the bacterial surface (for a review see [3-5]). They have a glycosylated lipopeptide core that is variably modified by $O$-methylation and $O$-acetylation (Fig. 1). More precisely, the GPL structure is based on a tripeptideaminoalcohol (D-Phe-D-allo-Thr-D-Ala-L-alaninol) Nlinked to a long chain fatty acyl residue. This lipopeptide core is substituted by a 6-deoxytalosyl (dTal) unit linked to the allo-Thr residue and by an $O$-methylated rhamnosyl unit linked to the terminal alaninol residue. The dTal residue can be $O$-acetylated on positions 3 and 4, further glycosylated on position 2 in M. avium, whereas the fatty acyl moiety and the rhamnosyl residue can be modified with one and three methyl groups on positions 2,3 , or 4 , respectively and in some cases further glycosylated by the addition of an extra rhamnosyl unit [3,11,6] (Fig. 1).

GPLs are required for sliding motility, biofilm formation and for maintaining cell wall integrity $[7,8]$. They also influence bacterial aggregation $[7,9]$, induce the release of prostaglandin E2 and interfere with the interaction between mycobacteria and human monocytes/macrophages $[10,11]$. Moreover, several recent studies show that natural variants of $M$. abscessus, which produce only small amounts of GPL are more invasive than the high-level producers [12-14]. Thus, GPLs appear to play an important role in both the physiology and the pathogenicity of mycobacteria.

The complete genomes of M. chelonae (CIP 104535T) and M. abscessus (CIP 104536T) have recently been sequenced to help to elucidate their molecular mechanisms of pathogenicity and antibiotic resistance. By exploiting available data concerning the genetic basis of the GPL biosynthetic pathway in the RGM model organism, M. smegmatis, we identified and analysed the genetic regions encoding enzymes involved in GPL biosynthesis in M. chelonae and M. abscessus.

\section{Results \\ Biochemical analysis of the glycopeptidolipid produced by M. abscessus and $M$. chelonae}

Since GPL molecules may differ at a structural level, we first assessed the GPL status of the strains used in this study for comparative genomic analysis: M. smegmatis $\mathrm{mc}^{2} 155$, M. chelonae CIP $104535 \mathrm{~T}$ and M. abscessus CIP 104536T. Strains were grown in both early exponential and late stationary phases (Fig. 2A) and lipids were extracted from cells [11]. The GPLs were identified by thin-layer chromatography (TLC), a method separating molecules according to their hydrophobic index, and using matrix-assisted laser desorption/ionization time of flight (MALDI-TOF) mass spectrometry analysis [11]. Since GPLs contain sugar moieties, GPL-like compounds were identified by anthrone staining [7]. Whatever the growth phase, the three strains produced similar diglycosylated GPLs, and in comparable quantities; however, triglycosylated GPLs were clearly more abundant in $M$. chelonae and M. abscessus than in M. smegmatis in both conditions (Fig. 2A). The precise nature of the GPL-like compounds was resolved by MALDI-TOF mass spectrometry, a very accurate and sensitive method for detecting glycolipids. The molecular masses of all the GPL-associated pseudomolecular ions peaks detected in the mass spectra of all strains perfectly matched the previously calculated molecular weights of the GPLs in these strains. Most of the GPLs produced by M. smegmatis mc $^{2} 155$ were diglycosylated forms, confirming earlier reports [15]. M. abscessus and $M$. chelonae produced the same diglycosylated GPLs $([\mathrm{M}+\mathrm{K}+] \mathrm{m} / \mathrm{z}$ at $1273 \mathrm{amu})$, and in addition produced a higher amount of triglycosylated GPLs than M. smegmatis $([\mathrm{M}+\mathrm{K}+] \mathrm{m} / \mathrm{z}$ at $1419 \mathrm{amu})$ as expected from TLC analysis (Fig. 2B).

\section{Comparative genomics of the GPL biosynthetic pathway}

Comparative genomic analysis was performed by pairwise alignments of the proteins of the GPL locus of M. smegmatis and each of the complete proteomes of M. abscessus and $M$. chelonae $[16,17]$. All the genes necessary for GPL biosynthesis are clustered in a single region of the M. smegmatis genome, thus constituting a true "GPL locus" (Figure 3). In this species, most of the genes of the locus (Fig. 1) have been identified experimentally and/or by in silico prediction (Table 1). The tripeptide-aminoalcohol moiety is non-ribosomally assembled by the product of the mps 1 and the mps 2 genes $[18,19]$, and the lipid synthesis and attachment to the tripeptide-aminoalcohol moiety probably require the concerted action of several gene products (pks, fadD23, papA3) [19,20]. The genes involved in the glycosylation of the lipopeptide core $(g t f 1, g t f 2)$ were recently characterized by selectively inactivating them both and by biochemical analysis of the resulting mutants [21]. Triglycosylated GPLs, described as being produced mostly during stationary phase [22], result from the addi- 


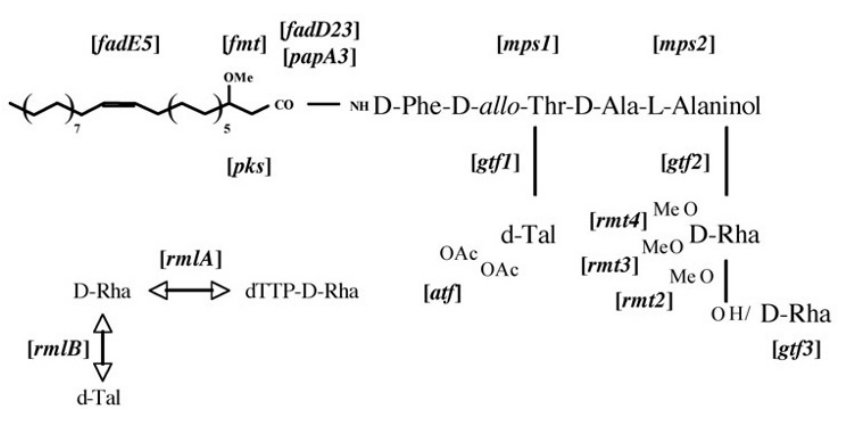

Figure I

Schematic representation of the structure of the GPLs from M. smegmatis. The genes involved in the various metabolic steps are indicated in brackets. The locations of the variable substitutions linked to the common GPL core are shown. OAc: acetyl, OMe, methyl. dTal, 6-deoxytalose, D-Rha, rhamnose of the $D$ series.

tion of an extra rhamnosyl residue ( $g t f 3)[9,21]$. The genes involved in the $O$-methylation of the various hydroxyl groups of the rhamnosyl unit (rmt2, rmt3 and rmt4) and of the lipid moiety $(\mathrm{fmt})$ of the GPLs have been characterized using similar methods [23-25]. The atf gene product is responsible for acetylating two particular hydroxyl residues of the dTal [26], and FadE5 probably introduces the double bond into the fatty acid. The $r m l A$ and $r m l B$ genes are associated with the activation and epimerisation of the sugars [27]. Two members of the $m m p L$ family, a group of genes encoding large membrane proteins, are also required for GPL biosynthesis $[19,26]$. The exact function of these proteins, MmpL4a and MmpL4b, is not known in detail although a recent study performed in M. tuberculosis suggests that MmpL proteins (MmpL7) may channel the polyketide products during their synthesis by the polyketide synthase, coupling synthesis and export [28]. Finally, the transport of the GPLs to the surface of the bacilli involves the integral membrane protein Gap [19]. This locus also contains eight other genes that have not yet been experimentally characterized: two encode membrane proteins ( $m m p S 4$ and $m m p L 10$ ) that may also interact with the polyketide synthase of the cluster, two encode a sigma factor and a sigma-associated protein (ecf and sap, respectively) that are believed to contribute to the regulation of GPL production, and four have no known function (Table 1 and Fig. 1) $[18,19]$.

All the genes of the M. smegmatis GPL locus have close orthologs in both M. chelonae and M. abscessus (Table 1). These orthologs share more than $80 \%$ of identity with each other and most are more than $90 \%$ identical (data not shown). The percentage of identity between $M$. chelonae/M. abscessus and M. smegmatis orthologs ranges between 30 and $89 \%$, with two-thirds of $M$. chelonae/M. abscessus orthologs being $\geq 70 \%$ identical to their M. smegmatis counterparts. Identity is less than $50 \%$ for only four orthologs: sap, ecf, Rv0926 and Rv1174c. The functions of $R v 0926$ and $R v 1174 c$ are not known, sap and ecf may play roles in the regulation of GPL biosynthesis, and were this the case, it would suggest that the regulatory circuits in these species have diverged.

Unlike M. smegmatis, the M. chelonae/M. abscessus GPL orthologs are not gathered in a single region (Figure 3). In both $M$. chelonae and M. abscessus, there is a large region containing 19 genes ( $m m p S 4$ to gap). This region contains all genes involved in the synthesis of the tripeptide-aminoalcohol moiety, the glycosylation of the lipopeptide and the $\mathrm{O}$-methylation and $\mathrm{O}$-acetylation modifications (see also Fig. 1). This region is very similar to the corresponding region of the M. smegmatis GPL locus, except for the two following differences. First, there is no mobile element, either upstream of $m b t H$ like in M. smegmatis $\mathrm{mc}^{2} 155$ or at any other location. Second, there are two atf orthologs (we called them atf1 and atf2) in both $M$. chelonae and M. abscessus: atf1 is at the same location as atf in M. smegmatis whereas atf2 is inserted between $r m l B$ and rmt2. M. chelonae atf1 and atf 2 genes are $58 \%$ identical ( $71 \%$ similarity), and are 76 and $60 \%$ identical to $M$. smegmatis atf respectively (88 and $75 \%$ similarity respectively); $M$. abscessus atf 1 and atf 2 genes are $57 \%$ identical (72\% similarity), and are 72 and 59\% identical to atf respectively ( 83 and $74 \%$ similarity respectively). There is a smaller region forming a block of 6 genes $900 \mathrm{~kb}$ from this first region in M. chelonae and $3 \mathrm{Mb}$ away in M. abscessus. These six genes ( $p k s$ to gap-like) are probably involved in the lipid synthesis and attachment to the tripeptideaminoalcohol moiety (e.g., pks, fadD23, papA3), but pks is the only one that has been experimentally studied so far [19]. This block is part of a large region that is inverted between M. chelonae and M. abscessus. It is very similar to the corresponding part of the M. smegmatis GPL locus except that the order of the pe and the fadD23 genes is switched in M. chelonae/M. abscessus relative to M. smegmatis. Finally, four genes closely linked in M. smegmatis (Rv0926, fadE5, sap, ecf) are scattered on the chromosome in $M$. chelonae and M. abscessus, with distances differing between

species.

To test whether the locus organization was a particularity of the sequenced strains (CIP 104535T and CIP 104536T), 5 clinical isolates of each species were analyzed by PCR using two couples of primers (Additional file 1). All the M. abscessus and M. chelonae isolates had the same PCR pattern. This experiment shows that the genetic organization of the GPL locus depicted in Figure 3 is not strain-dependant but is probably valid for the whole species. 

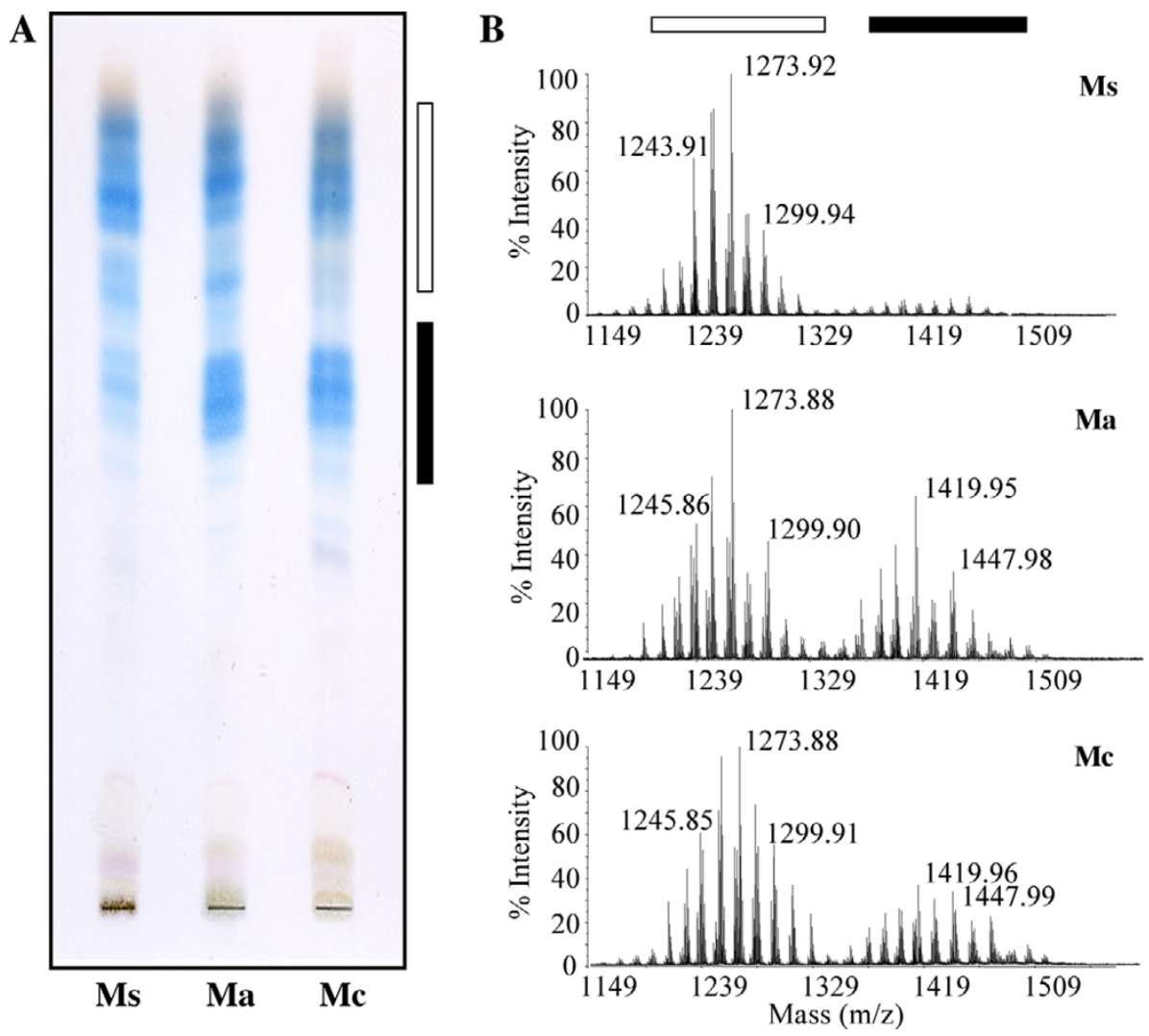

\section{Figure 2}

A) Thin-layer chromatography analysis of the crude lipid extract of M. smegmatis (Ms), M. abscessus (Ma), and M. chelonae (Mc). Open rectangle, diglycosylated GPLs; closed rectangle, triglycosylated GPLs. B) MALDI-TOF mass spectra of the crude lipid fractions of M. smegmatis (Ms), M. abscessus (Ma), and M. chelonae (Mc). Open rectangle, diglycosylated GPLs; closed rectangle, triglycosylated GPLs.

\section{Acetyltransferases have evolved specificity in M. abscessus and $M$. chelonae}

As seen above, there is only one atf in M. smegmatis while there are two in both $M$. abscessus and M. chelonae. In $M$. smegmatis, Atf catalyses the transfer of two acetyl groups onto the dTal moiety (on positions 3 and 4) [26]. We consequently tested whether the presence of the 2 atf in the other species was a redundant or specialization process and used atf1 and atf2 of M. abscessus as a model. We used a M. smegmatis atf- mutant as a recipient host and complemented it with constructs expressing the various atf genes (Additional file 2). The GPLs produced by the various complemented strains were analysed by both TLC and MALDI-TOF. The GPLs produced by the M. smegmatis atfmutant were, as expected, non $\mathrm{O}$-acetylated $([\mathrm{M}+\mathrm{K}+] \mathrm{m} / \mathrm{z}$ at 1189 and $1215 \mathrm{amu}$ ) as deduced from the value of the masses of the pseudomolecular ions and its altered migration on TLC. Reintroduction of the atf gene of M. smegmatis led to the production of $\mathrm{O}$-diacetylated GPL $([\mathrm{M}+\mathrm{K}+]$ $\mathrm{m} / \mathrm{z}$ at 1273 and $1299 \mathrm{amu}$ ) with a wild type migration pattern. Complementation by the atf1 gene of M. abscessus was enabling the production of mono-O-acetylated GPL $([\mathrm{M}+\mathrm{K}+] \mathrm{m} / \mathrm{z}$ at 1231 and $1258 \mathrm{amu})$ having an intermediary migration between the di-O-acetylated and the nonacetylated forms, indicating a specialisation process. Surprisingly, the GPL produced by the M. smegmatis atfmutant complementated by the atf2 gene of $M$. abscessus was most exclusively non-acetylated as judged by TLC (Additional file 2) and MALDI-TOF analysis confirmed molecular masses of 1189 and 1215 amu (data not shown). However, when both atf1 and atf2 were simultaneously introduced into the host strain, the production of di-O-acetylated GPLs was restored as judged by TLC (Additional file 2) and MALDI-TOF analysis confirmed molecular masses of 1273 and 1299 amu (data not shown). This set of experiments shows that the atf2 gene of $M$. abscessus is fully functional and needs a mono-Oacetylated dTal substrate to be able to transfer the second acetyl moiety. In conclusion, the acetyltransferases encoded by the M. abscessus GPL locus are not redundant 
Table I: Genes of the M. smegmatis GPL locus and their orthologs in M. abscessus and M. chelonae.

\begin{tabular}{|c|c|c|c|c|c|c|}
\hline \multicolumn{3}{|r|}{ M. smegmatis } & \multicolumn{2}{|c|}{ M. abscessus } & \multicolumn{2}{|c|}{ M. chelonae } \\
\hline Gene & Gene $n^{\circ}$ & Proposed function (a) & Gene $n^{\circ}$ & $\%(b)$ & Gene $n^{\circ}$ & $\%(b)$ \\
\hline$m m p S 4$ & MSMEG0373 & Membrane associated. Interaction with $\mathrm{Pks},{ }^{\circ}$ & ma4II7c & 78 & $\mathrm{mcl} 618$ & 74 \\
\hline$m m p L 4 a$ & MSMEG0374 & Membrane associated. Interaction with $\mathrm{Pks}{ }^{\circ}$ & $\mathrm{ma} 41 \mathrm{I} 6 \mathrm{c}$ & 78 & $\mathrm{mcl} 619$ & 77 \\
\hline$m m p L 4 b$ & MSMEG0375 & Membrane associated. Interaction with Pks, ${ }^{\circ}$ & ma4II5c & 76 & $\mathrm{mcl} 620$ & 75 \\
\hline$R v / I 74$ & MSMEG0376 & None & ma4II4 & 48 & $\mathrm{mcl} 62 \mathrm{lc}$ & 48 \\
\hline$r m l A$ & MSMEG0377 & Alpha-D-hexose-I-phosphate-thymidylyl-transferase (EC 2.7.7.24), ${ }^{\circ}$ & $\mathrm{ma} 4 \mathrm{II} 3$ & 85 & $\mathrm{mcl} 622 \mathrm{c}$ & 85 \\
\hline gtf3 & MSMEG0378 & D-Rhamnose rhamnosyltransferase, + & ma4II2c & 49 & $\mathrm{mcl} 623$ & 71 \\
\hline$r m / B$ & MSMEG0379 & UDP-hexose 4-epimerase, ${ }^{\circ}$ & ma4IIIc & 77 & $\mathrm{mcl} 624$ & 78 \\
\hline$r m t 2$ & MSMEG0380 & Rhamnose 2-O-methyltransferase, + & ma4109c & 72 & $\mathrm{mcl} 626$ & 70 \\
\hline$r m t 4$ & MSMEG038I & Rhamnose 4-O-methyltransferase, + & ma4l08c & 83 & $\mathrm{mcl} 627$ & 83 \\
\hline gtfl & MSMEG0382 & D-allo-threonine 6-deoxytalosyltransferase, + & ma4107c & 76 & $\mathrm{mcl} 628$ & 77 \\
\hline \multirow[t]{2}{*}{ atf } & MSMEG0383 & Integral membrane protein. 6-deoxytalose 3,4-O-acetyltransferase, + & $\mathrm{ma} 4106 c^{(c)}$ & 72 & $\mathrm{mcl} 629(\mathrm{c})$ & 76 \\
\hline & & & ma4l I0c(d) & 59 & $\mathrm{mcl} 625(\mathrm{~d})$ & 60 \\
\hline$r m t 3$ & MSMEG0384 & Rhamnose 3-O-methyltransferase, + & ma4105c & 81 & $\mathrm{mcl} 630$ & 82 \\
\hline gtf & MSMEG0385 & L-alaninol rhamnosyltransferase, + & ma4I04 & 67 & $\mathrm{mcl} 63 \mathrm{lc}$ & 72 \\
\hline fmt & MSMEG0386 & Fatty acid O-methyltransferase, + & $\operatorname{ma} 4103 c$ & 67 & $\mathrm{mcl} 632$ & 62 \\
\hline$m b t H$ & MSMEG0387 & None & ma4100c & 89 & $\mathrm{mcl} 635$ & 89 \\
\hline mpsl & MSMEG0390 & Non-ribosomal protein synthase. Synthesis of the dipeptide, $+^{\circ}$ & $\operatorname{ma} 4099 \mathrm{c}$ & 70 & $\mathrm{mcl} 636$ & 70 \\
\hline mps2 & MSMEG0392 & Non-ribosomal protein synthase. Synthesis of the amino acid alcohol, $+^{\circ}$ & $\operatorname{ma} 4098 c$ & 71 & $\mathrm{mcl} 637$ & 72 \\
\hline gap & MSMEG0393 & Integral membrane protein. Required for GPL export, + & $\operatorname{ma} 4097 \mathrm{c}$ & 58 & $\mathrm{mcl} 638$ & 55 \\
\hline sap & MSMEG0394 & Sigma-associated protein & $\operatorname{ma} 4454 c$ & 30 & $\mathrm{mcl} 299$ & 30 \\
\hline ecf & MSMEG0395 & Sigma factor of the ECF family. Required for regulation & $\operatorname{ma} 4459 c$ & 46 & $\mathrm{mcl} 294$ & 47 \\
\hline fadE5 & MSMEG0396 & Fatty acid desaturase, ${ }^{\circ}$ & ma4437 & 78 & $\mathrm{mcl} 318 \mathrm{c}$ & 78 \\
\hline Rv0926 & MSMEG0397 & None & $\mathrm{ma} 4633$ & 36 & $\mathrm{mcl} 136 \mathrm{c}$ & 36 \\
\hline pks & MSMEG0398 & Fatty acid synthesis and activation, $+^{\circ}$ & ma0939 & 79 & mc0819 & 79 \\
\hline рарАЗ & MSMEG0399 & Transfer of the Pks-bound fatty acid to the pseudotetrapeptide, ${ }^{\circ}$ & $\mathrm{ma0938c}$ & 77 & $\mathrm{mc} 0818 \mathrm{c}$ & 77 \\
\hline$m m p L I 0$ & MSMEG0400 & Membrane associated. Interaction with Pks, ${ }^{\circ}$ & $\mathrm{ma0937c}$ & 75 & $\mathrm{mc} 0817 \mathrm{c}$ & 74 \\
\hline fadD23 & MSMEG040I & Long chain fatty acyl-AMP ligase, ${ }^{\circ}$ & $\mathrm{ma0935c}$ & 73 & $\mathrm{mc} 0815 \mathrm{c}$ & 74 \\
\hline pe & MSMEG0402 & None & $\mathrm{ma0936c}$ & 64 & $\mathrm{mc} 0816 \mathrm{c}$ & 66 \\
\hline gap-like & MSMEG0403 & Integral membrane protein. Role in the transport of GPLs, ${ }^{\circ}$ & $\mathrm{ma0934}$ & 55 & $\mathrm{mc} 0814$ & 55 \\
\hline
\end{tabular}

(a) + , experimentally validated function; ${ }^{\circ}$, in silico predicted function.

(b) Percentage of identity between $M$. abscessus or M. chelonae genes and M. smegmatis genes.

(c) atfl (see text and legend to Fig. 3).

(d) atf2 (see text and legend to Fig. 3)

but have evolved specificity, being able to transfer one acetyl at once in a sequential manner.

\section{Discussion}

This study is the first addressing the genetics of GPL biosynthesis in two clinically significant RGM species, M. chelonae and $M$. abscessus. The major observation is that, despite producing structurally identical GPL molecules, the genes necessary for its biosynthesis are organized very differently. In M. smegmatis, the GPL locus is made up of almost 30 genes in a region of $\sim 65 \mathrm{~kb}$, and therefore does not comply with the prokaryotic rule of 1 gene $/ \mathrm{kb}$. This is because GPL biosynthesis involves very large multi-modular proteins, for example the non-ribosomal protein synthetases (Mps1 and Mps2) and the polyketide synthase (Pks), and consequently very long genes. Several genes appear to be organized into operons, one of which has been identified formally and contains $m b t H, m p s 1, m p s 2$, gap, sap and ecf [19]. Interestingly, a mobile element, IS1096, is located just upstream from $m b t H$ in $\mathrm{mc}^{2} 155$ strain. This upstream region corresponds to the promoter of the $m b t H$ operon and may therefore interfere with the expression of this operon, as it does in other biological systems $[29,30]$. Surprisingly, M. chelonae and M. abscessus produced clearly more triglycosylated GPL than M. smegmatis. This observation argues in favour of differences in gtf3 expression in these three species.

All the genes are clustered in M. smegmatis, but are scattered in several blocks in $M$. chelonae and M. abscessus. The various genomic pieces correspond to blocks of function: one block corresponds to the synthesis of the tripeptideaminoalcohol moiety, the glycosylation of the lipopeptide and $\mathrm{O}$-methylation/acetylation modifications, and another to lipid biosynthesis and its attachment to the tripeptide-aminoalcohol moiety. In addition, these species differ by one inversion and one duplication. An attractive hypothesis is that the compact organization of the GPL locus in M. smegmatis represents the ancestral form and that evolution has scattered various pieces throughout the genome in M. abscessus and M. chelonae. However, the opposite hypothesis in which genes involved in a metabolic pathway would have the tendency to gather during evolution cannot be excluded. The fact that both $M$. chelonae and M. abscessus have two nonredundant $O$-acetyltransferases suggests that atf 2 may 

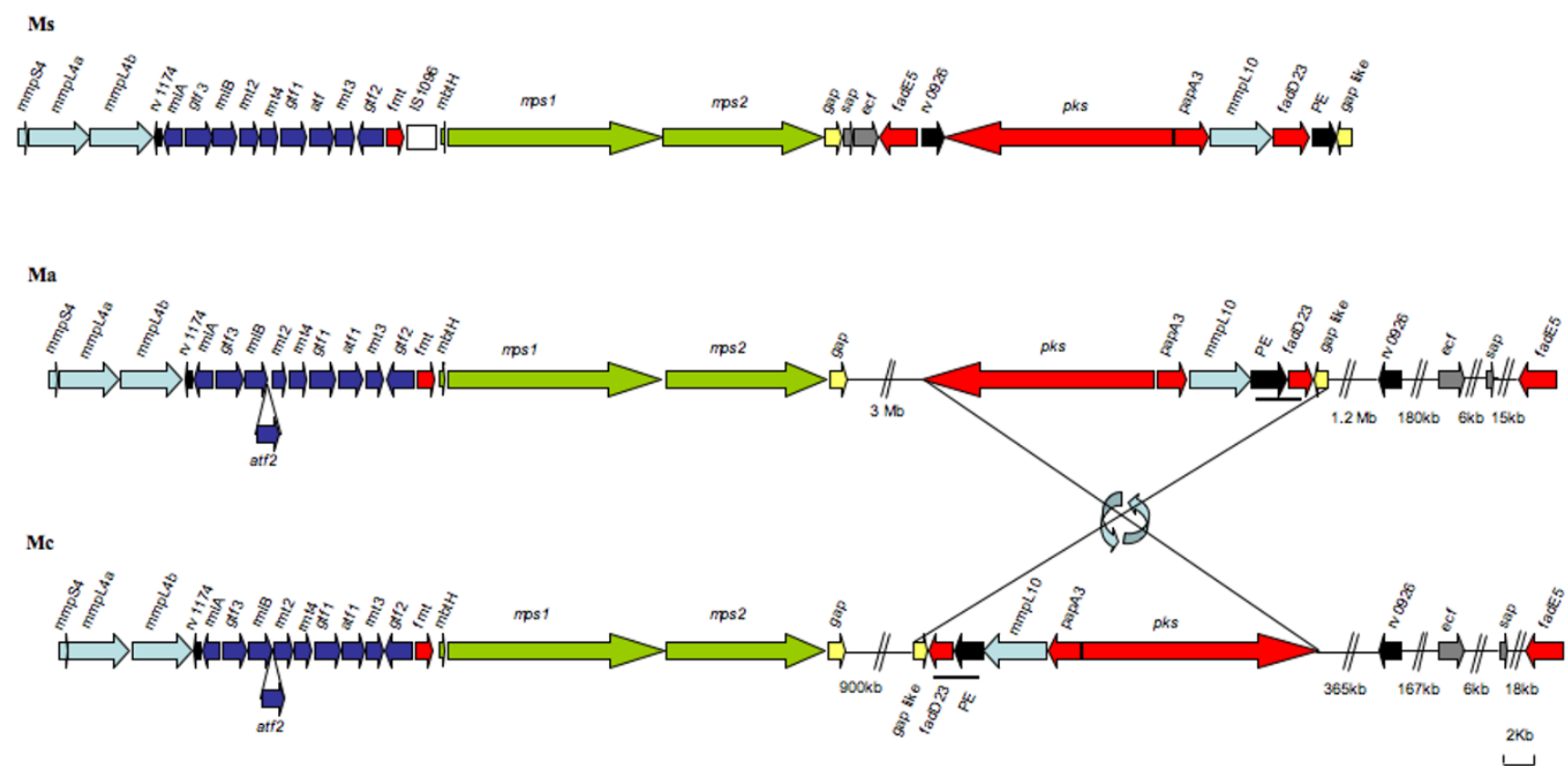

\section{Figure 3}

Genetic organization of the GPL locus in M. smegmatis (Ms), M. abscessus (Ma), and M. chelonae (Mc). The ORFs are depicted as arrows and have been drawn to scale. Colour code: Light blue: $m m p L$ family, black: unknown, purple: sugar biosynthesis, activation, transfer and modifications, red: lipid biosynthesis, activation, transfer and modifications, green: pseudopeptide biosynthesis, yellow: required for GPL transport to the surface, grey: regulation.

have arisen from the duplication of atf1. Interestingly, atf1 is less similar to atf 2 than to M. smegmatis atf, also indicating a functional divergence between atf1 and atf2. In $M$. smegmatis, atf mediates the $O$-acetylation of the dTal in both positions 3 and 4. We showed that, in M. abscessus, atf 1 and atf 2 are each specifically responsible for one of these two reactions and that probably act sequentially.

We showed that the GPL biosynthetic pathway is highly conserved between $M$. chelonae and $M$. abscessus, consistent with the close relatedness of these two species [31]. However, due to genomic rearrangements between the two species, the two blocks are located at different coordinates and the block of six genes is inverted in these two species with respect to that in M. smegmatis. These genomic rearrangements are consistent with the separation of the two species that were formerly parts of a single complex [31]. We showed, using a panel of clinical isolates that these differences are species-specific, and may thus be used as a discriminative marker. The genomic findings are in agreement with the biochemical data showing that the two species produce structurally identical GPL molecules [3]. However, differences in terms of regulation cannot be excluded and it is not known whether additional genes are needed for GPL biosynthesis, export and regulation in these three species.

M. chelonae and M. abscessus, like other mycobacterial species $[32,33]$, can naturally switch from a rough (R) to smooth (S) and from a $S$ to a $R$ morphotype $[12,13]$. $R$ strains are associated with a low GPL production, high invasive ability and a higher virulence in the mouse model $[13,14]$. However, despite several attempts, the genetic bases for this natural $S / R$ switching remain obscure. Several studies using M. avium and M. smegmatis describe various genes involved in the transition between $\mathrm{S}$ and $\mathrm{R}$ morphotypes, most of which are implicated in the GPL biosynthetic pathway $[18,19,34]$. The identification of the genes required for the synthesis and export of these metabolites should help our understanding of the natural variation in the morphology and virulence variation of these species.

\section{Conclusion}

We showed that $M$. abscessus and M. chelonae contain genes corresponding to all those of the M. smegmatis "GPL locus" with an extensive conservation of the predicted protein sequences. This finding is consistent with the pro- 
duction of GPL molecules indistinguishable by either thin-layer chromatography or mass spectrometry. Despite, the genomic and structural homology, the GPL locus appears to be split into several parts in $M$. chelonae and M. abscessus. One large cluster (19 genes) comprises all genes involved in the synthesis of the tripeptide-aminoalcohol moiety, the glycosylation of the lipopeptide and $\mathrm{O}$-methylation/acetylation modifications. A second smaller and distant (M. chelonae, $900 \mathrm{~kb} ;$ M. abscessus, 3 $\mathrm{Mb})$ cluster of six genes is involved in the synthesis of the fatty acyl moiety and its attachment to the tripeptide-aminoalcohol moiety. The other genes are scattered throughout the genome, including two genes encoding putative regulatory proteins. Although these three species produce identical GPL molecules, the organization of GPL genes differs between them, thus constituting species-specific signatures. An attractive hypothesis is that the compact organization of the GPL locus in M. smegmatis represents the ancestral form and that evolution has scattered various pieces throughout the genome in $M$. abscessus and $M$. chelonae, although the opposite scenario cannot be excluded.

\section{Methods \\ Bacterial strains}

M. smegmatis $\mathrm{mc}^{2} 155$ and $M$. abscessus CIP104536T (ATCC 19977T) were cultured in 7H9 supplemented with $10 \% \mathrm{ADC}$ at $37^{\circ} \mathrm{C}$. M. chelonae CIP 104535T (ATCC $35752 \mathrm{~T}$ ) was cultured in the same medium at $30^{\circ} \mathrm{C}$. All bacterial cultures were harvested in either early exponential or late stationary phase. When required, antibiotics were included at the following concentrations: kanamycin, $50 \mu \mathrm{g} / \mathrm{ml}$, hygromycin, $200 \mu \mathrm{g} / \mathrm{ml}$ (for E. coli) or 50 $\mu \mathrm{g} / \mathrm{ml}$ (for mycobacteria).

\section{Lipid analysis}

Lipids were extracted from cells with a mixture of chloroform and methanol and further partitioned by methanol precipitation as previously described [11]. The GPLs (250 $\mu \mathrm{g}$ lipid each deposit) were identified by TLC on silica gel Durasil 25-precoated plates (Macherey-Nagel) run in chloroform/methanol (90:10 [vol/vol]) and using MALDI-TOF mass spectrometry analysis [11]. These sugarcontaining compounds were identified by spraying plates with $0.2 \%$ anthrone in concentrated sulfuric acid, followed by heating at $110^{\circ} \mathrm{C}$ [7].

\section{Computer methods}

The accession number of the GPL locus of M. smegmatis is AY439015. The sequencing and the assembly of the genome of M. abscessus and M. chelonae was performed by the CNS (Centre National de Séquençage-Evry-France), [35]. Open reading frames of both $M$. abscessus and $M$. chelonae were predicted using both SHOW [36] and ARTEMIS [37]. The accession numbers corresponding to the regions of the GPL locus of M. abscessus are AM31616 to AM31621. The accession numbers corresponding to the regions of the GPL locus of M. chelonae are AM231610 to AM231615. The complete sequence of M. abscessus and M. chelonae will be reported elsewhere (J. L Risler \& J. L Gaillard, unpublished data). The 6901 putative proteins of the genome of $M$. smegmatis were obtained from The Institute for Genomic Research [38]. The comparative genomic analysis was performed by pairwise alignments between the proteins of the GPL locus of M. smegmatis and each of the complete proteomes mentioned above. These comparisons were performed using the LASSAP software and $Z$-values were calculated as described $[16,17]$. The identification of the orthologous links was performed using the results of the pairwise comparisons as follows: For each gene of the GPL locus of M. smegmatis, 5 bi-directional best hits $(\mathrm{BBH})$ were identified. The $\mathrm{BBH}$ having the best Z-value was selected. When several BBH exhibited a similar Z-value (some of the genes of the GPL locus such a $f a d D$ and $f a d E$ are affected by a high degree of paralogy), the gene preserving the syntenic context was selected. Identity below $25 \%$ was not considered as significant. All the selected orthologs have a Z-value greater than 14 (except the sap gene and its orthologs).

\section{Analysis of the M. abscessus and M. chelonae clinical isolates using PCR}

The chromosomal DNA was prepared using the beadbeater-phenol extraction method. The bacterial pellet (corresponding to $50 \mathrm{ml}$ culture) were suspended in $5 \mathrm{ml}$ of solution I (25\% sucrose; $50 \mathrm{mM}$ TrisCl $1 \mathrm{M} \mathrm{pH}=8 ; 50$ $\mathrm{mM}$ thiourea; $10 \mathrm{mg} / \mathrm{ml}$ lysozyme). The thiourea inhibits the Tris-dependent DNAse that is present in some strains [39]. Solution II (25\% sucrose; $50 \mathrm{mM}$ TrisCl $\mathrm{pH}=8 ; 50$ $\mathrm{mM}$ EDTA $\mathrm{pH}=8$ ) was added and the bacterial cells were lysed as described by Howard and al. [40]. Proteinase K was added to the lysate at $100 \mu \mathrm{g} / \mathrm{ml}$ and incubated overnight at $55^{\circ} \mathrm{C}$. The DNA was extracted using phenol/chlorophorm/isoamyl-alcohol $(25: 24: 1)$ and precipitated with propanol. Primers (mpsF1, mpsF2, mpsR; pkF1, pkF2, pkR) (Additional file 3) were designed according to the chromosomal sequence of $M$. abscessus and $M$. chelonae. PCR amplification was performed using Dynazyme Taq polymerase according to manufacturer instructions (Finnzyme, Espoo, Finland).

\section{Construction of acetyltransferase expression plasmids}

The wild type $M$. smegmatis atf gene (accession number AY138899) coding sequence was amplified by PCR using the Pfu DNA Polymerase (Stratagene), the genomic DNA of M. smegmatis mc $^{2} 155$ as template and primers containing an engineered $\mathrm{XbaI}$ site (atfsmeg.5 and atfsmeg.3) (Additional file 3). After purification with the PCR purification Qiagen kit, PCR products were digested with XbaI and cloned into the dephosphorylated expression vector 
pNIP40b [41] at the unique XbaI site to generate pNIPatfsmeg. One clone having the atfsmeg gene inserted in the opposite direction of the hygromycin resistant gene was selected and sequenced. Using M. abscessus ATCC $19977^{\mathrm{T}}$ genomic DNA as template, a similar strategy was applied to clone atf1 gene and atf2 gene (AM231618) using primers atf1abs.5/atf1abs.3 and primers atf2abs.5/atf2abs.3 (Additional file 3) into pNIP40b [41] yielding pNIPatf1absc and pNIPatf2absc, respectively. To clone the $M$. abscessus atf1 and atf2 genes in frame, the af1absc PCR product was digested by ClaI and the atf2 gene was amplified using new primers (atf2ClaI.5 and atf2abs.3) and digested with ClaI. The 2 PCR products were digested with $X b a \mathrm{I}$, purified and ligated, with the dephosphorylated expression vector pNIP40b at its unique XbaI site to generate pNIPatf1_2absc. These plasmids were electroporated into M. smegmatis $\mathrm{mc}^{2} 155$ atf- mutant [26] and transformants were selected on plates containing kanamycin and hygromycin. These strains are named atf-/atfsMs, atf-/ atf1Ma, atf-/atf2Ma and atf-/atf1_2Ma.

\section{Abbreviations}

\section{GPL: glycopeptidolipid}

RGM: rapidly growing mycobacteria

dTal: 6-deoxytalose

D-Rha: rhamnose of the D series

TLC: thin-layer chromatography

MALDI-TOF: matrix-assisted laser desorption/ionization time-of-flight

amu: atomic mass unit

\section{Authors' contributions}

FR carried out the bioinformatic studies, analysed the Ma and Mc strains by PCR and drafted the manuscript. CD carried out the molecular biology experiments, participated in the sequence alignment and drafted the manuscript. SP participated in the sequence alignment. FL and JLB produced and analysed the MS data. FB collected and cultivated various $M$. avium subsp. avium strains used as internal control and helped to draft the manuscript. JLR participated in the sequence alignments and in the phylogenetic analysis. MD participated in the analysis of the biochemical experiments. JLG participated in the design of the study and helped to draft the manuscript. GE performed biochemical experiments, participated in the design of the study and helped to draft the manuscript. JMR conceived of the study, participated in its design and coordination and drafted the manuscript. All authors read and approved the final manuscript.

\section{Additional material}

\section{Additional File 1}

Analysis of various clinical isolates of $\mathrm{M}$. abscessus and $\mathrm{M}$. chelonae $b y$ PCR.

Click here for file

[http://www.biomedcentral.com/content/supplementary/1471-

2164-8-114-S1.tiff]

\section{Additional File 2}

A) Thin-layer chromatography analysis of the crude lipid extracts of wildtype M. smegmatis (1), the atf- mutant (2), the atf- mutant complemented by the atf gene of $\mathrm{M}$. smegmatis (3), the atf1 (4) or atf2 (5) genes of M. abscessus or both (6). B) MALDI-TOF mass spectra of the crude lipid fractions of the various $\mathrm{M}$. smegmatis atf complemented strains.

Click here for file

[http://www.biomedcentral.com/content/supplementary/14712164-8-114-S2.tiff]

\section{Additional File 3}

List of the oligonucleotides used in this study. Click here for file

[http://www.biomedcentral.com/content/supplementary/14712164-8-114-S3.doc]

\section{Acknowledgements}

Data were obtained from TIGR from their website at [38]. The M. smegmatis genome was sequenced with support of the NIAID. We thank R. Kolter for kindly providing the $M$. smegmatis atf- strain. We acknowledge Inserm for funding this project under the Avenir programme to JMR, Chargé de Recherches at Inserm. FR is supported by the association "Vaincre la Mucoviscidose". CD is funded by a doctoral grant of Fondation pour la Recherche Médicale (FRM). We thank D. Fogg for English correction of the manuscript.

\section{References}

I. Brown-Elliott BA, Wallace RJ Jr.: Clinical and taxonomic status of pathogenic nonpigmented or late-pigmenting rapidly growing mycobacteria. Clin Microbiol Rev 2002, I5(4):716-746.

2. Sanguinetti M, Ardito F, Fiscarelli E, La Sorda M, D'Argenio P, Ricciotti G, Fadda G: Fatal pulmonary infection due to multidrugresistant Mycobacterium abscessus in a patient with cystic fibrosis. J Clin Microbiol 200I, 39(2):8I 6-8I9.

3. Billman-Jacobe $\mathrm{H}$ : Glycopeptidolipid synthesis in Mycobacteria. Current Science 2004, 86: II-I I4.

4. Daffe $M$ and Lemmassu A.: Glycobiology of the mycobacterial surface. Structures and biological activities of the cell enveloppe glycoconjugates. In Glycomicrobiology Edited by: Doyle . New-York, Kluwer Academic / Plenum Publishers; 2000:225-273.

5. Brennan PJ: Mycobacterium and other actinomycetes. In Microbial Lipids Volume I. Edited by: Ratledge CWSG. London, UK , Academic Press; 1988:203-298.

6. Lopez-Marin LM, Gautier N, Laneelle MA, Silve G, Daffe M: Structures of the glycopeptidolipid antigens of Mycobacterium abscessus and Mycobacterium chelonae and possible chemical basis of the serological cross-reactions in the Mycobacterium fortuitum complex. Microbiology 1994, I40 ( Pt 5): I I09- III 8.

7. Etienne G, Villeneuve C, Billman-Jacobe H, Astarie-Dequeker C, Dupont MA, Daffe M: The impact of the absence of glycopeptidolipids on the ultrastructure, cell surface and cell wall properties, and phagocytosis of Mycobacterium smegmatis. Microbiology 2002, I 48(Pt I 0):3089-3। 00. 
8. Recht J, Martinez A, Torello S, Kolter R: Genetic analysis of sliding motility in Mycobacterium smegmatis. J Bacteriol 2000, I 82( I 5):4348-435I.

9. Deshayes C, Laval F, Montrozier H, Daffe M, Etienne G, Reyrat JM: A Glycosyltransferase Involved in Biosynthesis of Triglycosylated Glycopeptidolipids in Mycobacterium smegmatis: Impact on Surface Properties. I Bacteriol 2005, I 87(2I):7283-729I.

10. Barrow WW, Davis TL, Wright EL, Labrousse V, Bachelet M, Rastogi $\mathrm{N}$ : Immunomodulatory spectrum of lipids associated with Mycobacterium avium serovar 8. Infect Immun 1995 63(I):126-133.

II. Villeneuve C, Etienne G, Abadie V, Montrozier H, Bordier C, Laval F, Daffe M, Maridonneau-Parini I, Astarie-Dequeker C: Surfaceexposed glycopeptidolipids of Mycobacterium smegmatis specifically inhibit the phagocytosis of mycobacteria by human macrophages. Identification of a novel family of glycopeptidolipids. J Biol Chem 2003, 278(5 I):5 I29I-5 I300.

12. Byrd TF, Lyons CR: Preliminary characterization of a Mycobacterium abscessus mutant in human and murine models of infection. Infect Immun 1999, 67(9):4700-4707.

13. Howard ST, Rhoades E, Recht J, Pang X, Alsup A, Kolter R, Lyons CR, Byrd TF: Spontaneous reversion of Mycobacterium abscessus from a smooth to a rough morphotype is associated with reduced expression of glycopeptidolipid and reacquisition of an invasive phenotype. Microbiology 2006, I52(Pt 6): I58|-|590.

14. Catherinot E, Clarissou J, Etienne G, Ripoll F, Emile JF, Daffe M, Perronne C, Soudais C, Gaillard JL, Rottman M: Hypervirulence of a rough variant of the Mycobacterium abscessus type strain. Infect Immun 2007, 75(2): 1055- 1058.

15. Etienne G, Laval F, Villeneuve $C$, Dinadayala $P$, Abouwarda A, Zerbib $D$, Galamba A, Daffe M: The cell envelope structure and properties of Mycobacterium smegmatis $\mathrm{mc}(2)$ 155: is there a clue for the unique transformability of the strain? Microbiology 2005, I 5 I (Pt 6):2075-2086

16. Comet JP, Aude JC, Glemet E, Risler JL, Henaut A, Slonimski PP, Codani J]: Significance of Z-value statistics of Smith-Waterman scores for protein alignments. Comput Chem 1999, 23(34):3|7-33|.

17. Glemet E, Codani J]: LASSAP, a LArge Scale Sequence compArison Package. Comput Appl Biosci 1997, I3(2): I37-I 43.

18. Billman-Jacobe H, McConville M], Haites RE, Kovacevic S, Coppel RL: Identification of a peptide synthetase involved in the biosynthesis of glycopeptidolipids of Mycobacterium smegmatis. Mol Microbiol I999, 33(6): I244-I 253.

19. Sonden B, Kocincova D, Deshayes C, Euphrasie D, Rhayat L, Laval F, Frehel C, Daffe M, Etienne G, Reyrat JM: Gap, a mycobacterial specific integral membrane protein, is required for glycolipid transport to the cell surface. Mol Microbiol 2005, 58(2):426-440.

20. Trivedi OA, Arora P, Sridharan V, Tickoo R, Mohanty D, Gokhale RS Enzymic activation and transfer of fatty acids as acyl-adenylates in mycobacteria. Nature 2004, 428(698I):44I-445.

21. Miyamoto Y, Mukai T, Nakata N, Maeda Y, Kai M, Naka T, Yano I, Makino M: Identification and characterization of the genes involved in glycosylation pathways of mycobacterial glycopeptidolipid biosynthesis. J Bacteriol 2006, I 88(I):86-95

22. Mukherjee R, Gomez M, Jayaraman N, Smith I, Chatterji D: Hyperglycosylation of glycopeptidolipid of Mycobacterium smegmatis under nutrient starvation: structural studies. Microbiology 2005, I 5 I (Pt 7):2385-2392.

23. Jeevarajah D, Patterson JH, McConville MJ, Billman-Jacobe H: Modification of glycopeptidolipids by an O-methyltransferase of Mycobacterium smegmatis. Microbiology 2002, I48(Pt 10):3079-3087.

24. Jeevarajah D, Patterson JH, Taig E, Sargeant T, McConville MJ, Billman-jacobe $\mathrm{H}$ : Methylation of GPLs in Mycobacterium smeg. matis and Mycobacterium avium. I Bacteriol 2004, 186(20):6792-6799

25. Patterson JH, McConville MJ, Haites RE, Coppel RL, Billman-Jacobe H: Identification of a methyltransferase from Mycobacterium smegmatis involved in glycopeptidolipid synthesis. J Biol Chem 2000, 275(32):24900-24906.

26. Recht J, Kolter R: Glycopeptidolipid acetylation affects sliding motility and biofilm formation in Mycobacterium smegmatis. J Bacteriol 200I, I 83(19):57|8-5724.
27. Cole ST, Brosch R, Parkhill J, Garnier T, Churcher C, Harris D, Gordon SV, Eiglmeier K, Gas S, Barry CE 3rd, Tekaia F, Badcock K, Basham D, Brown D, Chillingworth T, Connor R, Davies R, Devlin K, Feltwell T, Gentles S, Hamlin N, Holroyd S, Hornsby T, Jagels K, Barrell BG: Deciphering the biology of Mycobacterium tuberculosis from the complete genome sequence. Nature 1998, 393(6685):537-544.

28. Jain M, Cox JS: Interaction between Polyketide Synthase and Transporter Suggests Coupled Synthesis and Export of Virulence Lipid in M. tuberculosis. PLoS Pathog 2005, I(I):e2.

29. Hammerschmidt S, Hilse R, van Putten JP, Gerardy-Schahn R, Unkmeir A, Frosch M: Modulation of cell surface sialic acid expression in Neisseria meningitidis via a transposable genetic element. Embo J 1996, I5(I): 192-198.

30. Poussier S, Thoquet P, Trigalet-Demery D, Barthet S, Meyer D, Arlat $M$, Trigalet A: Host plant-dependent phenotypic reversion of Ralstonia solanacearum from non-pathogenic to pathogenic forms via alterations in the phcA gene. Mol Microbiol 2003, 49(4):991-1003.

31. Kusunoki S, Ezaki T: Proposal of Mycobacterium peregrinum sp. nov., nom. rev., and elevation of Mycobacterium chelonae subsp. abscessus (Kubica et al.) to species status: Mycobacterium abscessus comb. nov. Int J Syst Bacteriol 1992, 42(2):240-245

32. Barrow WW, Brennan PJ: Isolation in high frequency of rough variants of Mycobacterium intracellulare lacking C-mycoside glycopeptidolipid antigens. I Bacteriol 1982, I50(I):38I-384.

33. Belisle JT, Klaczkiewicz K, Brennan PJ, Jacobs WR Jr., Inamine JM: Rough morphological variants of Mycobacterium avium. Characterization of genomic deletions resulting in the loss of glycopeptidolipid expression. J Biol Chem 1993, 268(14): 10517-10523.

34. Laurent JP, Hauge K, Burnside K, Cangelosi G: Mutational analysis of cell wall biosynthesis in Mycobacterium avium. J Bacteriol 2003, 185(16):5003-5006

35. Genoscope projects [http://www.cns.fr/externe/English/Projets/]

36. Bioinformatic Tools [http://migale.jouy.inra.fr/outils/ select all outils zpt]

37. Rutherford K, Parkhill J, Crook J, Horsnell T, Rice P, Rajandream MA Barrell B: Artemis: sequence visualization and annotation. Bioinformatics 2000, 16(1 0):944-945.

38. J. Craig Venter Institute [http://www.tigr.org/]

39. Zhang Y, Yakrus MA, Graviss EA, Williams-Bouyer N, Turenne C, Kabani A, Wallace RJ Jr.: Pulsed-field gel electrophoresis study of Mycobacterium abscessus isolates previously affected by DNA degradation. J Clin Microbiol 2004, 42( ( 2):5582-5587.

40. Howard ST, Byrd TF, Lyons CR: A polymorphic region in Mycobacterium abscessus contains a novel insertion sequence element. Microbiology 2002, I 48(Pt I 0):2987-2996.

4I. Vultos TD, Mederle I, Abadie V, Pimentel M, Moniz-Pereira J, Gicquel B, Reyrat JM, Winter N: Modification of the mycobacteriophage Ms6 attP core allows the integration of multiple vectors into different tRNAala T-loops in slow- and fast-growing mycobacteria. BMC Mol Biol 2006, 7:47.

Publish with Biomed Central and every scientist can read your work free of charge

"BioMed Central will be the most significant development for disseminating the results of biomedical research in our lifetime. "

Sir Paul Nurse, Cancer Research UK

Your research papers will be:

- available free of charge to the entire biomedical community

- peer reviewed and published immediately upon acceptance

- cited in PubMed and archived on PubMed Central

- yours - you keep the copyright 